\title{
SPLITTING TERRACED HOUSES INTO SINGLE UNITS USING OBLIQUE AERIAL IMAGERY
}

\author{
D. Dahlke \\ ${ }^{\text {a } I n s t i t u t e ~ o f ~ O p t i c a l ~ S e n s o r ~ S y s t e m s, ~ D L R ~ B e r l i n, ~ G e r m a n y ~ d e n n i s . d a h l k e @ ~ d l r . d e ~}$
}

Commission II, WG II/4

KEY WORDS: Façade Splitting, Oblique True Ortho, Building Segmentation

\begin{abstract}
:
This paper introduces a method to subdivide complex building structures like terraced houses into single house units comparable to units available in a cadastral map. 3D line segments are detected with sub-pixel accuracy in traditional vertical true orthomosaics as well as in innovative oblique true orthomosaics and their respective surface models. Hereby high gradient strengths on roofs as well as façades are taken into account. By investigating the coplanarity and frequencies within a set of 3D line segments, individual cut lines for a building complex are found. The resulting regions ideally describe single houses and thus the object complexity is reduced for subsequent topological, semantical or geometrical considerations. For the chosen study area with 70 buidling outlines a hit rate of $80 \%$ for cut lines is achieved.
\end{abstract}

\section{INTRODUCTION}

The automated extraction of photorealistic $3 \mathrm{D}$ city models is an emerging task in the field of remote sensing. These models contain geometric information like building borders or street furniture, semantic information like storey numbers or window-towall ratios as well as textures from terrestrial or oblique aerial images. An established geographically referenced data model and exchange format is the official international OGC standard CityGML (Kolbe, 2009) with certain Level-of-Details (LoD). The subject of this paper is part of a workflow for automated extraction of textured and semantically annotated "LoD-2.5" CityGML objects (Frommholz et al., 2016) from aerial oblique imagery. At this LoD, buildings have distinctive roof structures, roof overhangs and façades with window information. Prior to this approach building outlines have been derived with contemporary algorithms (Piltz et al., 2016) from standard vertical true orthomosaics and their corresponding digital surface model. Figure 1(a) shows one of such polygons, which usually do not represent single houses, but the (roof) outline of terraced houses. An inherent part of reconstruction algorithms is to describe the world's complexity as simple but precise as possible. In order to achieve satisfying results on basis of the aforementioned building outlines, it is necessary to split them into smaller and semantically reasonable units. Otherwise topological and semantic derivations likely become too complex and irrational which yields in unrecoverable geometry for the buildings in question.

The presented approach splits complex polygons into reasonable units. It works on basis of the very same input data as mentioned above, namely surface models and true orthomosaics derived from triangulated vertical and oblique aerial imagery. An edge detection is performed for each orthomosaic within a building outline polygon (Figure 1(c)-(f)). All obtained line segments are shown in Figure 1(b). They are analysed with respect to their 3D orientation and local frequency. Hereby split lines as well as small gaps between buildings are introduced as 2D line segments spanning from one building outline border to another. Within the resulting smaller units a subsequent 3D building reconstruction part is much more robust against topological and geometric blunders. Another benefit for this further processing step is the consistency of input data.

Since the segmented building outline polygon is comparable to a cadastral map, a comparison between both is part of the experimental results section. Due to several reasons it is not advisable to rely on a cadastral map for the segmentation of complex building outlines. For one thing they don't compulsory match geographically to the acquired image data. This is due to either old cadastral maps with lower accuracies or geodetic datum shifts between different coordinate systems. Displacements in the range of decimetres between image and cadastre information may occur, which makes this combination ineffective. For another thing cadaster information is related to additional costs, since it is not open source if the data is available at all. Especially in fast growing cities, like Istanbul for example, with many terraced houses, it is not given to get maintained cadaster information for this purpose.

\subsection{Related work}

Basically there are two fusion possibilities for 2D polygonal databases like cadastral maps and 3D building models. Firstly 3D building models are taken for granted and the 3D reconstruction stage is not necessary. In this case the focus lays on the correct spatial join between existing 3D models and attributed 2D datasets which are commonly available in municipal administrations. Hereby an existing, visually appealing 3D building model inherits semantic information for enhanced spatial analysis. Moreover the challenge with this is that one building model could be related to many $2 \mathrm{D}$ datasets, therefore screw up citywide spatial analysis. In order to achieve a one-to-one relationship the geographic link between $2 \mathrm{D}$ and $3 \mathrm{D}$ dataset is used to create a new 3D building model, containing divided models for each entry in the cadastral database (Pedrinis and Gesquière, 2017). Urban planning companies like CyberCity3D even state that "Splitting 3D buildings is key for detailed 3D spatial analysis" (Hughes and Sullivan, 2015). An incorporation of façade characteristics from vertical imagery to improve unfitting cadaster information can be found in (Meixner et al., 2012). 
Secondly 2D databases are used as initial regions of interest (ROI) for 3D building reconstrunction from aerial images (Cohen and Vinson, 2002) or LIDAR pointclouds (Kada and McKinley, 2009). These initial polygons are either derived from the raw input data, representing the roof outlines (Mayer, 2002) or taken from municipal ground plans (Vosselman and Dijkman, 2001). All approaches have in common that they decompose initial complex ROIs into smaller, preferably rectangular, units in order to discriminate adjacent houses and simplify the reconstruction stage. The novelty of the presented method is the additional usage of true 3D information for façades from oblique imagery.

\subsection{Source data}

A flight campaign over the German islands of Heligoland was conducted using the DLR MACS HALE aerial camera (Bucher et al., 2016) with slightly more than $80 \%$ overlap along track. The two oblique sensors pointing to the left and right have a tilt angle of $36^{\circ}$. Firstly the exterior orientation for the cameras is approximated by filtering GPS/INS signals. Secondly an aerotriangulation is obtained by using the MATCH-AT module of INPHO. Finally for each ground pixel all corresponding acquisition geometries and their errors respectively matching costs are minimized (Hirschmüller, 2008). The result is a 2.5D digital surface model (DSM) projected in the ETRS89/UTM reference frame. The dense matching, which has been initially designed for the creation of 2.5D data from High Resolution Stereo Camera images, is applied to the oblique data as well. Both oblique camera heads of the MACS are triggered synchronously with the vertical camera head. An approach for generating oblique surface models for the extraction of geoinformation is presented in (Wieden and Linkiewicz, 2013). In contrast to traditional vertical DSM products, so called local reference frames (LRF) have to be defined beforehand. A LRF is described by a cluster of oblique images which have similar viewing geometries. In figures this means that rotation differences between images contributing to a cluster differ by no more than $20^{\circ}$. The mean of all contributing viewing angles is calculated and applied as sequential rotation angles to a rotation matrix. In total several clusters and rotation matrices are created for oblique imagery. Oblique surface models have similar properties like classical surface models derived from vertical aerial imagery. Each oblique surface model is geo referenced in a LRF. Because of the rotation parameters each LRF is connected to the higher level global reference frame (ETRS89/UTM) and therefore also to each other. Following this approach, the oriented oblique images are pre-processed into (tilted) DSMs. In case of a crossing stripes flight pattern, each occlusion free point in the scene is hereby mapped from different cardinal directions. The buildings are represented by at least five digital surface models and five orthomosaics respectively, one for the vertical case and at least four (e.g. each cardinal direction) for the oblique case. Due to the different viewing geometries, the ground sampling distance (GSD) differs between datasets. For the nadir case the GSD is $5 \mathrm{~cm}$. In the oblique case the GSD is roughly three times worth with $15 \mathrm{~cm}$.

Cadastral building boundaries serve as a reference for the proposed method. In Germany the land survey register contains among semantic, topologic and other geometric information the cadastral building footprints. They are delivered as polygon vector files projected in the ETRS89/UTM reference system. Depending on the survey method each building corner point possesses an error margin up to $6 \mathrm{~cm}$ (Vorschriftensammlung für das Vermessungswesen, 2016).

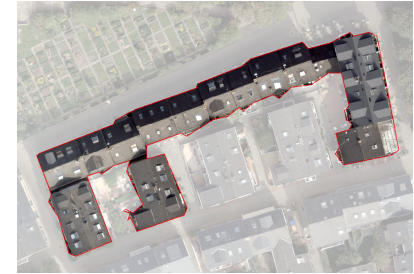

(a)

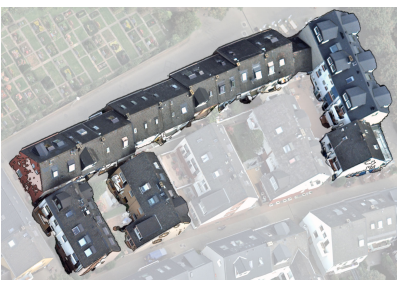

(c)

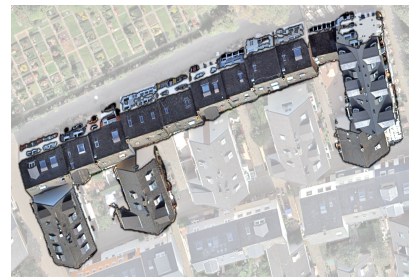

(e)

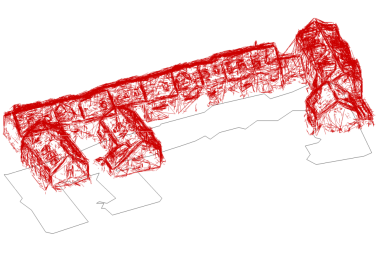

(b)

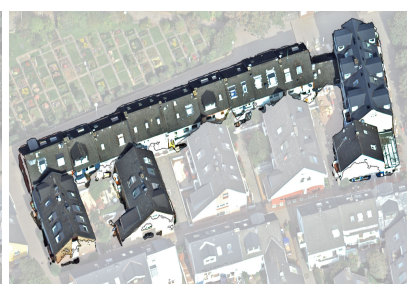

(d)

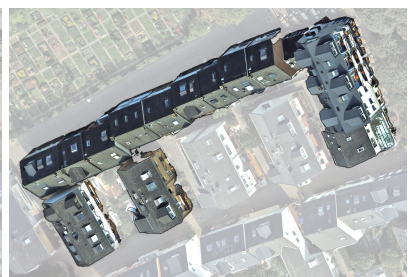

(f)
Figure 1. Source data (a) Vertical orthomosaic with superimposed building outline polygon (b) 31873 3D line segments from all orthomosaics (c-f) Oblique orthomosaics

showing the polygonal content as translucent parts

\section{PROPOSED METHOD}

Within traditional vertical true orthomosaics, single roofs are obviously distinguishable when they are made out of different materials. That means it can be well discriminated between red and black bricks. In addition firewalls, different roof textures, slopes or heights support the discrimination between different roofs. But the applicability of these roof features is not guaranteed, since they are not forcibly available. Façade features like drainage channels or differently colored walls provide additional support for discriminating different single house units within terraced houses. For façade interpretation the raw oblique imagery is processed analogous to the vertical aerial images as described in the previous section. 3D line segments are obtained from traditional vertical true orthomosaics as well as innovative oblique true orthomosaics and its respective surface models. For this purpose sub-pixel accurate algorithms like (Grompone von Gioi et al., 2012) and (Lu et al., 2015) can be used. Split lines are determined by investigating the coplanarity and frequencies within a set of 3D line segments. In addition to cut lines between terraced houses, the presented method also detects small gaps between physically divided houses.

\subsection{D line segments}

3D line segments are initially generated on all surface models and orthomosaics within the given building outline. For the detection of salient lines a fast line segment detector is used. Since this a gradient based image processing approach some important edges like a roof ridge cannot be detected in surface models as solely input. That is why true orthomosaices are derived from the surface models and used as additional inputs for the line segment detection. 


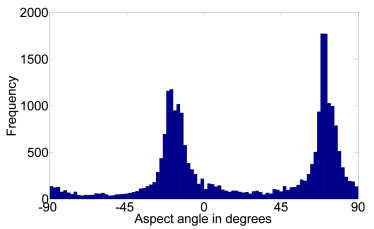

(a)

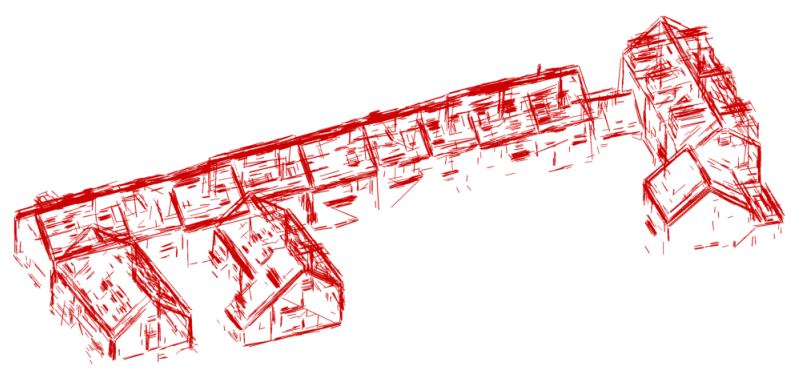

(c)

Figure 2. Filtering 3D line segments (a) Orientation of line segments in the xy-plane (with superimposed results of maxima detection) (b) 3D Orientation of line segments against the z-plane (with superimposed results of maxima detection) (c)

Final selection of 8891 3D line segments

The georeferenced 2D coordinates of both line segments endpoints are connected to the height information from the corresponding surface model. Hereby line segments are described with 3D coordinates for their endpoints in a local reference frame LRF. The relationship between all four LRF is mathematically described in terms of rotation matrices. All line segment coordinates are multiplied with the corresponding transposed rotation matrix to get into a higher level UTM reference frame. An exemplary visualization for 31873 of such detected line segments in the global reference frame is depicted in Figure 1(b). The line segment detection is very sensitive to noise caused by matching inaccuracies in the surface model and artefacts in the orthomosaics. To overcome this drawback the principle 3D orientation for a building complex is estimated by analysing the frequency for attitudes of all segments. Attitude in this case means the orientation of a line segment in $3 \mathrm{D}$ space that is given on the one hand as horizontal alignment or aspect for façades and on he other hand as vertical alignment or slope for roofs. The ordering of start and endpoints for line segments is of no importance in this case. That means the aspect ranges from $-90^{\circ}$ to $90^{\circ}$, with $-90^{\circ}$ and $90^{\circ}$ being east-west aligned and $0^{\circ}$ being northsouth aligned, while the slope ranges from $0^{\circ}$ to $90^{\circ}$, with $0^{\circ}$ being horizontal and $90^{\circ}$ being vertical. Predominant horizontal and vertical alignments are derived from the $3 \mathrm{D}$ orientation of all line segments. Histograms for both angles can be found in Figure 2(a)-(b). They give a first impression of the distribution of predominant angles in the line segments. While the aspect analysis takes all segments into account, the slope analysis is confined solely to segments which have a predominant aspect angle. Since vertical and horizontal slope angles are inherently the most prominent features in the slope histogram, the first and last $10^{\circ}$ are cut off, so that the range is limited from $10^{\circ}$ to $80^{\circ}$. Within the histograms for aspect and slope, a polynomial function of degree 2 is fitted to the peaks. A delta of approximately $90^{\circ}$ between two identified aspect angles indicates a good outcome, since most çades are built orthogonally. The maxima are found to be the principle orientation of a building. Line segments which differ

from this principle orientation are omitted. All remaining line segments are supposed to represent real edges without significant noise influence. For the example complex from Figure 1(b) almost one quarter, namely 8891 line segments remain for the subsequent and final decomposition stage (see Figure 2(c)).

\subsection{Decomposition at gaps and adjacent houses}

Initial outline polygons usually do not represent single houses, but the outline of terraced houses. Sometimes it also happens that gaps between physically divided buildings are not present in these polygons. This occurs on the one hand in small shaded areas between buildings where no reliable height information is available. And on the other hand it happens that nearby trees become part of the building outline, although the near infrared channel is used to differentiate between elevated vegetation or building objects. Within a CityGML or similar model, however, a building object generally is supposed to be a single house. Thus, an outline has to be split into smaller units, ideally representing single houses. A split line is considered to be a straight line from one side of the outline segment to another, splitting a complex polygon into smaller units.

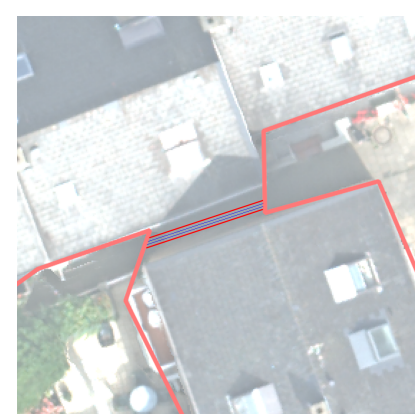

(a)

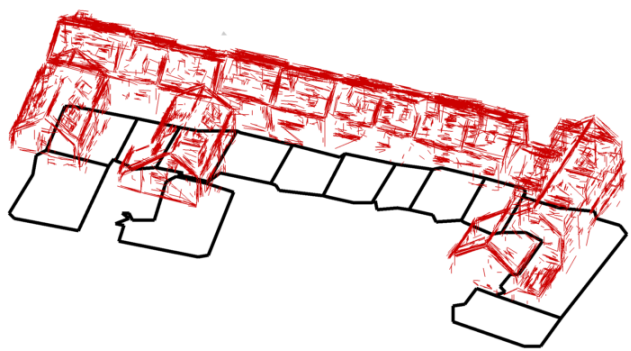

(c)

Figure 3. Decomposed building outlines (a-b) Gaps (blue slices) between physically divided houses with new borders (red slices) for initial (pink) building outline (c) Decomposed building polygon (black) based on 3D line segments (red)

After the pre-processing steps like line segment detection and filtering, the initial polygon is split into $2 \mathrm{D}$ slices. Step size and width of the slices is adapted to the ground sampling distance of the vertical input imagery. In the presented case this means in figures $10 \mathrm{~cm}$ for width and step size, which is twice the given GSD. The orientation of the slices is similar to the principle horizontal orientations of a building and perpendicular to the so called skeleton of the corresponding building polygon. Each centre of a slice is a possible candidate for a cut line, because a slice virtually already cuts the polygon into two pieces. In order to find valid cut 
lines, each slice is ranked by its containing line segments. Since slices usually do not contain complete 3D line segments, linearly spaced 3D points are generated for each segment. The number of evenly spaced points between the segment's endpoints depend on the segment's 3D extent. The GSD is used as distance for the spacing. As a result the projection of the $3 \mathrm{D}$ points onto the $\mathrm{xy}$ plane can be used for counting quantities and thereby measuring partial relationship between a slice and its containing line segments. Furthermore each 3D point still possesses the segment's original attributes like an ID or the 3D orientation. In the following these attributes and the quantity will be used to define cut lines.

Two contrary types of cut lines can be traced with these slices. In the aforementioned case of physically divided buildings, a slice contains almost no line segments. Within small shaded areas between buildings no line segments are detected at all. Figure 3(a) shows such a gap in a shaded area between two buildings. In the less usual case of high vegetation between two buildings (see Figure 3(b)), the orientation of initial line segments is arbitrary and therefore they are almost completely filtered out in the preprocessing stage. Neighbouring slices, containing no significant amount of line segments, are omitted and the building outline is split at the borders of this gap.

The second and by far more typical case is represented by slices containing many coplanar line segments. Basically this is the inverted case, compared to the aforementioned gap between physically divided houses. This time not only the presence but also the $3 \mathrm{D}$ orientation attribute of line segments is taken into account. Only if vertical and non vertical coplanar line segments are found coincidentally within one slice, the polygon is split at the centre of this slice. If many neighbouring slices fulfil this criteria, the centre of these adjacent slices is used as split line. An example for a decomposed complex building can be found in figure 3(c). An assumption, which both cut line types have in common is that the area of decomposed polygons has to be larger than $20 \mathrm{~m}^{2}$. The resulting split polygons roughly represent estate boundaries and are therefore comparable to official cadastral maps.

\section{RESULTS AND DISCUSSION}

For the study area, shown in the Appendix section, 70 initial building outlines have been used. From this starting point the presented approach detected 75 split lines. By applying these split lines to the initial 70 polygons, 136 segmented polygons were generated. The number of polygons has almost been doubled. Ten split lines are describing the borders of five gaps between physically divided buildings. The remaining 65 split lines are describing cut lines within building complexes. Compared to the corresponding cadastral map containing 75 complex buildings and 184 single house units (see Figure 5 in the Appendix section), four cut lines are false positives or false alarms. That means a splitting edge is introduced although no split is made in the cadastral map. Nevertheless these four lines are subdividing complex buildings with individual roof and façade structures. Furthermore 43 split lines have been missed, when compared against the cadastral map. With 71 true positive split lines and 43 false negatives, the sensitivity or hit rate is $62 \%$. However if taken into account, that almost 25 of the missed cut lines are not represented by any geometric or radiometric feature in the input datasets, the hit rate increases up to $80 \%$. In other words, the absence of those 25 undetected cut lines has no influence on the success of the subsequent reconstruction stage.

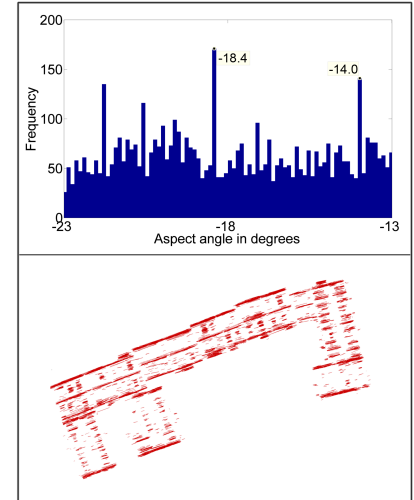

(a)

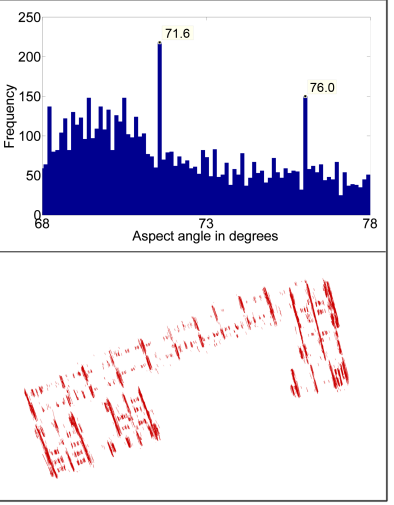

(b)
Figure 4. Different aspect angles within complex buildings (a-b) Subset of the aspect angle histogram with side maxima (top) and corresponding $3 \mathrm{D}$ line segments (bottom)

Another error shows up, when looking into the orientation angles of cut lines for complex buildings. If a complex building is composed out of single houses with different principle orientations, the split line orientation tends to be two or three degrees off. This is due to the fact that the orientation is derived from the maxima of the aspect angle histogram as shown in Figure 2(a). The top of Figure 4(a)-(b) shows a close-up of the aforementioned maxima regions. When picking manually the two strongest peaks within each region, it can be seen that the chosen orientation originally consists out of two different orientation angles. Each of the manually selected peaks has its counterpart for building up almost perfectly $90^{\circ}$. The visualisation of the corresponding line segments at the bottom of Figure 4(a)-(b) confirm the observed characteristic with slightly different orientated line segments for the easternmost building part.

\section{CONCLUSIONS AND OUTLOOK}

A novel approach to separate building footprints using vertical and oblique aerial images was presented. The main goal is to support a subsequent 3D reconstruction stage, which uses the very same input imagery. Based on building outline polygons a decomposition into single house segments was aspired, which serves as a step to reduce the complexity of the contour polygons. In a gradient based analysis of oblique and vertical aerial images, split line candidates were set up within initial building outlines. The final selection of split lines was performed by defining quality measures like observable coplanarity and frequency of 3D line segments. This way simple split lines from one outline border to another, are extracted. In order to determine the principle orientation of a building correctly, it is suggested to execute the proposed procedure iteratively. Firstly the detection of gaps between adjacent houses and secondly the splitting of terraced houses. All in all the approach identifies split lines for complex building footprints and makes use of façade and roof features like different construction materials or drainage channels. As reference for evaluation the cadaster dataset for the study area has been used. With respect to these reference lines an $80 \%$ hit rate is achieved by the automatically derived split lines. Taking into account, that these are the first findings and false positive line segments even support a subsequent $3 \mathrm{D}$ reconstruction, while false negatives not necessarily lead to unrecoverable buildings, the presented approach is very promising. Nevertheless the option to 
automatically derive or update cadastral boundaries has to be investigated critically.

Another part of future work will be the correct or corrected orientation of split line segments. While the orientation is basically derived very accurate (portions of a degree), it is possible that slight offsets occur for more complex buildings. An implementation for the detection and derivation of side maxima will probably lead to more reliable aspect orientations in this case. Since the orientation parameters of a building are derived from up to several thousand 3D line segments, they are, as already mentioned, for most of the buildings very accurate. This benefit could be perspectively used to support the 3D building reconstruction with reliable vertical and horizontal orientations for roofs and façades respectively.

\section{ACKNOWLEDGEMENTS}

Integration of the camera system and development of processing algorithms were done by the members of the Department for Sensor Concepts and Applications at the German Aerospace Centre. Representatively for the whole team I want to thank its team leaders Ralf Berger, Karsten Stebner, Sebastian Pless and Tilman Bucher. Without their innovative ideas neither such images would have been captured nor processed.

\section{REFERENCES}

Bucher, T., Berger, R., Brauchle, J., Hein, D., Lehmann, F., Piltz, B., Scherbaum, P., Stebner, K. and Pless, S., 2016. Modular photogrammetric sensor systems for real-time information extraction and 3d-applications. In: T. P. Kersten (ed.), 36. Wissenschaftlich-Technische Jahrestagung der DGPF, Publikationen der Deutschen Gesellschaft für Photogrammetrie, Fernerkundung und Geoinformation e.V., Vol. 25.

Cohen, L. D. and Vinson, S., 2002. Segmentation of complex buildings from aerial images and $3 \mathrm{~d}$ surface reconstruction. In: Proceedings of the Sixth IEEE Workshop on Applications of Computer Vision, WACV '02, IEEE Computer Society, Washington, DC, USA, pp. 215-219.

Frommholz, D., Linkiewicz, M. and Poznanska, A.-M., 2016. Inlining $3 \mathrm{~d}$ reconstruction, multi-source texture mapping and semantic analysis using oblique aerial imagery. In: L. Halounova, K. Schindler, A. Limpouch, T. Pajdla, V. Šafář, H. Mayer, S. O. Elberink, C. Mallet, F. Rottensteiner, M. Brédif, J. Skaloud and U. Stilla (eds), XXIII ISPRS Congress, The International Archives of the Photogrammetry, Remote Sensing and Spatial Information Sciences, Vol. XLInumber B3, Copernicus Publications, pp. 605612.

Grompone von Gioi, R., Jakubowicz, J., Morel, J.-M. and Randall, G., 2012. LSD: a Line Segment Detector. Image Processing On Line 2, pp. 35-55.

Hirschmüller, H., 2008. Stereo processing by semiglobal matching and mutual information. IEEE Trans. Pattern Anal. Mach. Intell. 30(2), pp. 328-341.

Hughes, T. and Sullivan, S., 2015. Splitting 3d buildings is key for detailed $3 \mathrm{~d}$ spatial analysis. https://cybercity3dglobal.wordpress.com/2015/11/02/splitting3d-buildings-is-key-for-detailed-3d-spatial-analysis/ (02 Nov. 2015).

Kada, M. and McKinley, L., 2009. 3d building reconstruction from lidar based on a cell decomposition approach. In: The International Archives of the Photogrammetry, Remote Sensing and Spatial Information Sciences, Vol. XXXVIII (3/W4), pp. 47-52.
Kolbe, T. H., 2009. Representing and exchanging 3d city models with citygml. In: J. Lee and S. Zlatanova (eds), $3 D$ GeoInformation Sciences, Lecture Notes in Geoinformation and Cartography, Springer, Berlin, Heidelberg, chapter 2, pp. 15-31.

Lu, X., Yao, J., Li, K. and Li, L., 2015. Cannylines: A parameterfree line segment detector. In: 2015 IEEE International Conference on Image Processing, ICIP 2015, Quebec City, QC, Canada, September 27-30, 2015, pp. 507-511.

Mayer, S., 2002. Gradient based splitting of blocks of houses in high-resolution ortho-images. In: Proceedings of the 2002 International Conference on Image Processing, ICIP 2002, Rochester, New York, USA, September 22-25, 2002, pp. 769-772.

Meixner, P., Wendel, A., Bischof, H. and Leberl, F., 2012. Building faade separation in vertical aerial images. ISPRS Annals of Photogrammetry, Remote Sensing and Spatial Information Sciences I-3, pp. 239-243.

Pedrinis, F. and Gesquière, G., 2017. Reconstructing 3D Building Models with the 2D Cadastre for Semantic Enhancement. Lecture Notes in Geoinformation and Cartography pp. 119 - 135.

Piltz, B., Bayer, S. and Poznanska, A.-M., 2016. Volume based $\mathrm{dtm}$ generation from very high resolution photogrammetric dsms. In: L. Halounova, K. Schindler, A. Limpouch, T. Pajdla, V. Šafár, H. Mayer, S. O. Elberink, C. Mallet, F. Rottensteiner, M. Brédif, J. Skaloud and U. Stilla (eds), ISPRS Congress, Vol. XLI-B3, pp. 83-90.

Vorschriftensammlung für das Vermessungswesen, 2016. http://www.stadtentwicklung.berlin.de/service/gesetzestexte/de/ download/geoinformation/Vorschriftensammlung/startseite.pdf.

Vosselman, G. and Dijkman, E., 2001. 3d building model reconstruction from point clouds and ground plans. Int. Arch. of Photogrammetry and Remote Sensing pp. 37-43.

Wieden, A. and Linkiewicz, M., 2013. True- obliqueorthomosaik aus schrägluftbildern zur extraktion von 3dgeoinformationen. In: DGPF Tagungsband, pp. 354-362. 

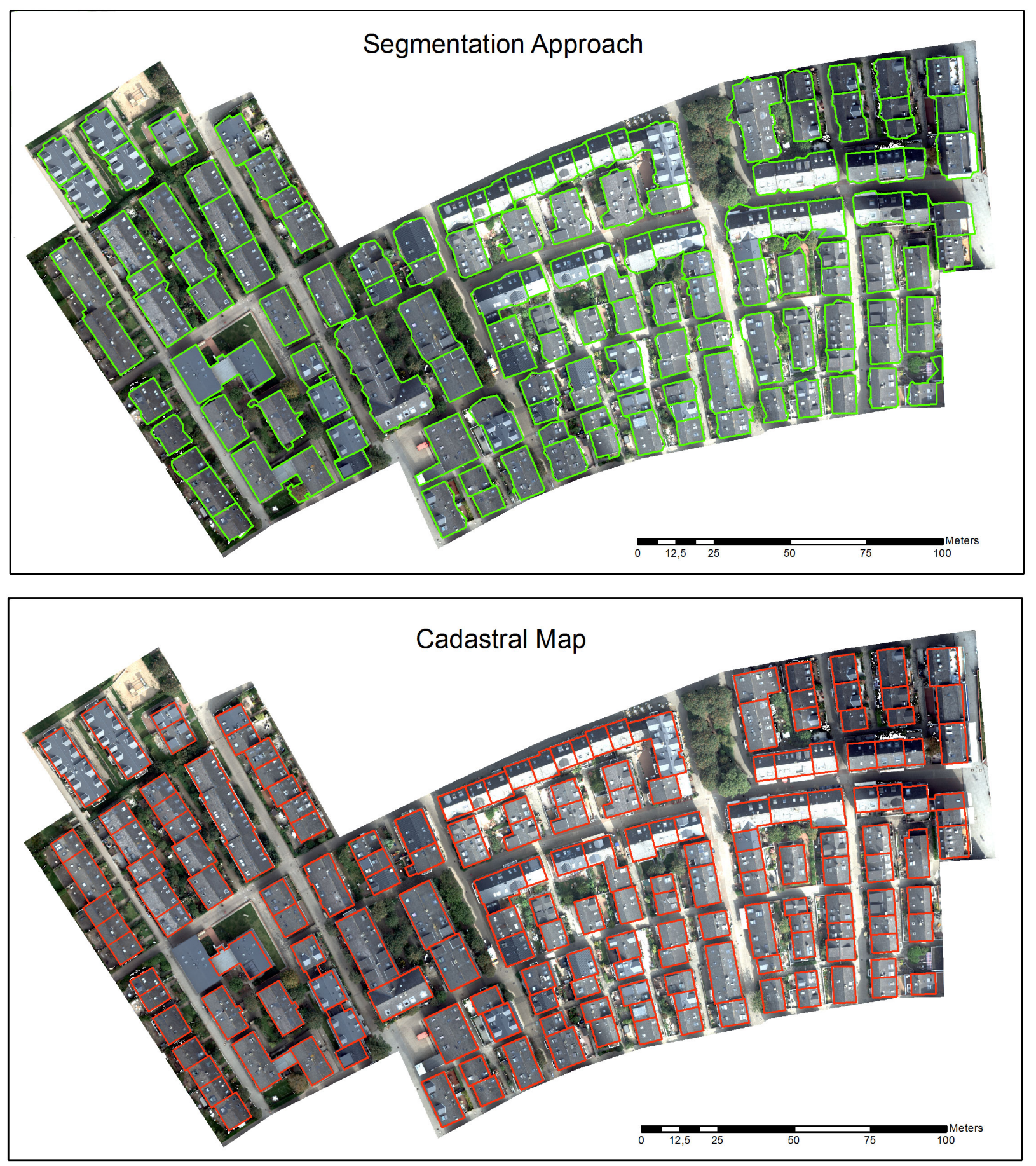

Figure 5. Result after the segmentation approach (top) vs. cadastral map (bottom) 